\title{
Refractive index matching (RIM) of liquid and semi-solid materials to acrylic glass for optically measuring the mechanics in soft granular matter
}

\author{
Lennart Vulprecht ${ }^{1}$ (1) $\cdot$ Christoph Gerstenberg ${ }^{1} \cdot$ Cornelia Rauh $^{1}$
}

Received: 25 March 2019 / Published online: 11 April 2020

(c) The Author(s) 2020

\begin{abstract}
The main context of this research is the fluid mechanical analysis of stirred chunky fruit preparations, which are typically highly loaded suspensions (ca. 50\%w/w) with particles susceptible to mechanical damage. Knowledge about the transport of such particles in fluid matrices is important in natural and technical processes and can be obtained using optical measurement techniques, e.g. Particle Image Velocimetry. Matching the refractive indices of the relevant material components, a way to ensure signal reliability, is difficult for highly concentrated dispersed systems. Material properties such as plasticity and elasticity of the solid phase and the rheological behaviour of the fluid must be met simultaneously. Fluid motion across the full range of the stirred volume and the immediate surroundings of the stirrer could not be observed without successful refractive index matching of acrylic glass, stirred liquid, and suspended particles. Using the presented materials, the mechanical firmness (but not the resistance against breaking) of soft granular matter can be mimicked. The movement of gel particles in suspensions, their resulting deformation and ultimately, the inflicted damage can be observed with optical methods. The rigidity of the gels may be varied to some extent with the concentrations of the respective hydrocolloids, which, at low concentrations, have no apparent effect on the refractive index. Introducing ethanol, thickeners or other components may yield more degrees of freedom in modelling their flow behaviour.
\end{abstract}

Keywords Particle Image Velocimetry (PIV) $\cdot$ Particle Tracking Velocimetry (PTV) $\cdot$ Fluid dynamics $\cdot$ Refractive index matching (RIM)

\section{Introduction}

\subsection{Background}

The main context of this research is the fluid mechanical analysis of stirred chunky fruit preparations. Fruit chunks can be described as solid matter particles which are vulnerable to mechanical influences. Typical products are highly loaded (ca. $50 \% \mathrm{w} / \mathrm{w}$ ) with particles and have a variety of available interactions such as flow-induced shear and strain and collisions with parts of the apparatus or with each other. Many flow problems, especially those involving particles,

Lennart Vulprecht

lennart.vulprecht@tu-berlin.de

1 Department of Food Biotechnology and Process Engineering, Technische Universität Berlin, Königin-Luise-Straße 22, 14195 Berlin, Germany are analyzed using a diversity of computational methods, e.g. the phenomena of jamming $[1,2]$ and deformability [3] in suspensions and emulsions. Experimental validation, because it is extremely difficult, sometimes receives little or no attention. The presented materials may be useful in such applications, especially, but not exclusively, for the modelling of biological materials. Possible examples include the study of jellyfish [4] and seaweed [5] in biology or blood clots and tissue blood flow in medicine [6].

Transport of particles in a fluid matrix is important in natural and technical processes and detailed knowledge of the fluid mechanics is therefore of high interest in food and other processing technologies like biotechnology [7-9]. It can be obtained using optical measurement techniques such as Laser Doppler Anemometry (LDA), Particle Tracking Velocimetry (PTV) and Particle Image Velocimetry (PIV). However, signal reliability of these techniques depends on several properties of the experimental setup. 


\subsection{Problem}

Systems analysed with optical methods need to be transparent to some extent. Refraction by the transparent materials involved can cause problems regarding both sufficient illumination of the flow and reliability of the signal. This is especially true for the investigation of multi-phase flows where refracting interfaces can be present anywhere in the control volume and are also very mobile. One approach to ensuring reliability of the results is to match the refractive indices of the relevant material components $[10,11]$. This is difficult for highly concentrated dispersed systems, which can also not be sufficiently investigated with invasive methods. Material properties such as plasticity and elasticity of the solid phase and the rheological behaviour of the fluid are crucial requirements to meet while simultaneously matching the refractive index of the necessarily rigid transparent material of the apparatus.

\subsection{Literature review}

Mixing and stirring technology are intensely studied subjects due to their importance in process engineering [12]. After the development of stirring technology from an empirical art to science [13], recent publications span across many disciplines, such as waste management [14], materials science [15] and renewable energy technology [16].

Particle Image Velocimetry (PIV) is a popular tool for the analysis of fluid mechanical phenomena. For a detailed description, refer to Particle Image Velocimetry: A Practical Guide [17]. Transparent materials are common in studying the mechanics in granular materials, e.g. soils [18]. Refractive index matching (RIM) can help improve the reliability of optical signals by selecting materials with the same or similar refractive indices. Model systems are sometimes developed to grant optical access while still behaving in a similar way to the original system. Some work has been published in this area concerning the solid materials used to model equipment [10, 19], application of different organic fluids $[19,20]$, and the use of ethanol and sodium chloride for the analysis of macroscopic stratified flows [21]. The latter is particularly interesting for matching a fluid phase to an acrylic glass apparatus [11], because at very high concentrations, the liquid reaches the same index as the acrylic glass. Hydrogels in general enable the transparent modelling of soft matter [22], flexible materials in the form of hydrogels made from polyacrylamide and agarose were made with refractive indices in the range of 1.33-1.35 [23], and refractive index matching of polyacrylamide has been described as a way to enhance the clarity of a hydrogel matrix for gel electrophoresis [24]. While synthetic hydrogels have been matched to water [25] to study geometrically complex flow obstacles and collagen has been used as a transparent matrix in which to study tumor and endothelial cells [26], a RIM approach, especially regarding PMMA, to modelling deformable, semi-solid particles mimicking biomaterials is missing in the literature and ongoing research on RIM in particle suspensions is mainly focused on small (microscopic) and rigid particles [27].

\section{Materials and methods}

\subsection{Stirring apparatus}

A stirring apparatus based on an industry design for the treatment of soft particle suspensions is modelled from transparent poly(methyl methacrylate), short: PMMA, acrylic glass or Plexiglas (brand name). It consists of a vessel with a tori-spherical bottom and an agitator (stirrer) of a modified anchor shape with three curved blades on each side (Fig. 1) and a thickness of $6 \mathrm{~mm}$. The material defines the refraction index (1.491) to which all other components are matched. This index is low compared to other transparent

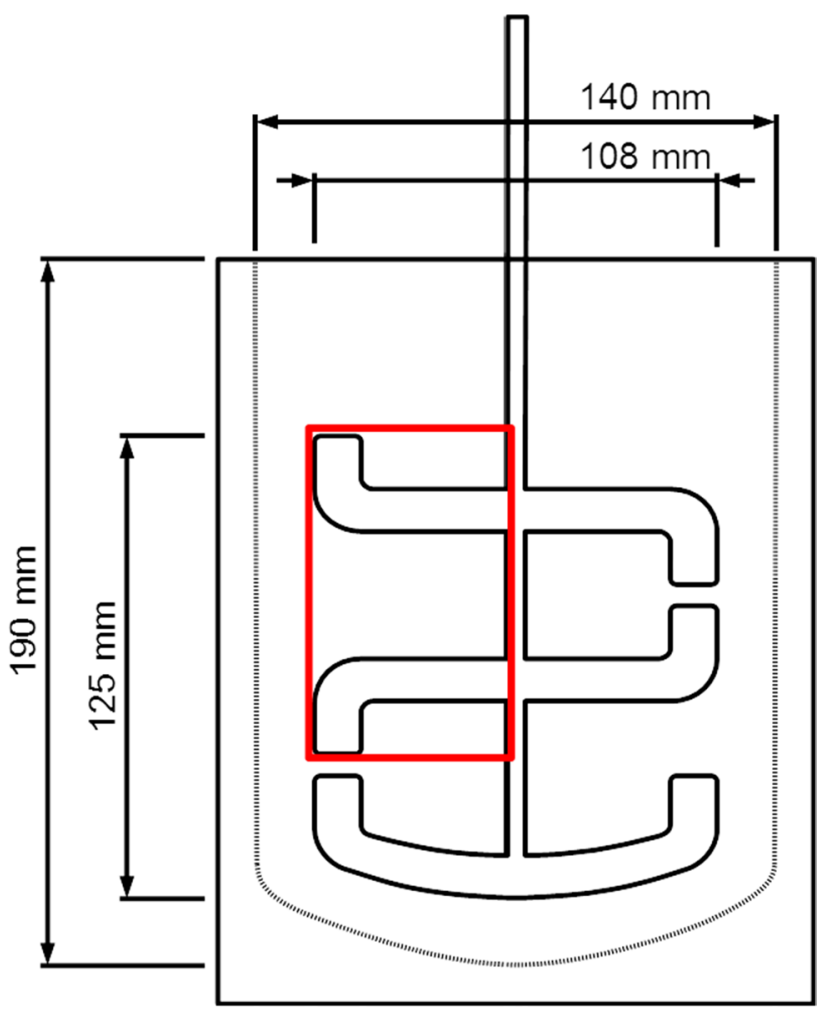

Fig. 1 Setup for stirring experiments, all parts shown made from transparent PMMA; the red rectangle marks the region of interest in test stirring experiments (color figure online) 
solids, e.g. crown glass: 1.517 or flint glass: 1.575 [28]. In addition, custom shapes are more easily available made from acrylic glass.

\subsection{Liquids}

A solution of $64 \% \mathrm{w} / \mathrm{w}$ sodium iodide $(\mathrm{NaI})$ in water has successfully been applied and reported on in the literature [11] as a matching fluid for PMMA. Temperature-dependent changes of viscosity are an essential factor in the fluid mechanics of suspensions. Because the refractive index changes with temperature as well [11], but not as strongly as the viscosity, it is easier to keep the temperature constant in the experiment. A change in viscosity then represents a change of temperature in the actual process. To increase the viscosity of the NaI solution, sucrose is added and the NaI concentration is lowered appropriately to keep the refractive index constant. This only works in a limited range, because the solution is not stable at sucrose concentrations above ca. $43 \% \mathrm{w} / \mathrm{w}$. Table 1 summarises the relevant material parameters for the involved liquids. Due to the high content of solutes, the two aqueous solutions have very high densities as compared to water $\left(\mathrm{ca} .1000 \mathrm{~kg} / \mathrm{m}^{3}\right.$ ) and saturated sucrose

Table 1 Dynamic viscosity, refractive index and density of RIM liquids measured at $20^{\circ} \mathrm{C}$

\begin{tabular}{llll}
\hline $\begin{array}{l}\text { Liquid (formula- } \\
\text { tion) }\end{array}$ & $\begin{array}{l}\text { Dynamic } \\
\text { viscosity } \\
(\mathrm{mPas})\end{array}$ & $\begin{array}{l}\text { Refrac- } \\
\text { tive index } \\
(\lambda=589 \mathrm{~nm})\end{array}$ & Density $\left(\mathrm{kg} / \mathrm{m}^{3}\right)$ \\
\hline $\begin{array}{l}64.0 \% \mathrm{w} / \mathrm{w} \mathrm{NaI} \\
36.0 \% \mathrm{w} / \mathrm{w} \text { Water }\end{array}$ & 3.5 & 1.495 & 1850 \\
$\begin{array}{l}42.4 \% \mathrm{w} / \mathrm{w} \text { NaI } \\
27.3 \% \text { w/w Sucrose }\end{array}$ & 23.7 & 1.497 & 1720 \\
$30.3 \% \mathrm{w} / \mathrm{w}$ Water & & & \\
\hline
\end{tabular}

solution (ca. $1400 \mathrm{~kg} / \mathrm{m}^{3}$ ). In the food industry, higher density usually coincides with higher viscosity.

Although refraction depends on the wavelength of light, it was only measured at the standard refractometer wavelength of $589 \mathrm{~nm}$. Red laser light $(650 \mathrm{~nm})$ as used in the following stirring experiments may be refracted differently. Table 2 lists the calculated refractive indices of materials used along with a few of the respective dispersion formulas. In conclusion, the relative change in refraction between the two wavelengths ranges from ca. $0.08 \%$ (water) to ca. $0.62 \%$ (NaI).

\subsection{Semi-solid particles}

Creating transparent, mechanically susceptible particles relies on the NaI solution introduced in the previous subsection. Because of the high salt content of $64 \% \mathrm{w} / \mathrm{w}$, which is just below saturation for $\mathrm{NaI}$, interactions of other solutes, especially gelling agents, are affected by the lack of disposable water. Hydrocolloid thickeners and combinations thereof allow the modelling of properties of biological materials. They are applied in microbiology (agar-agar) and food technology (carrageenans, locust bean gum). Experience shows that the texture of hydrocolloid gels, also called hydrogels, can be similar to that of biological materials. In this context, specifically the mechanical resistance, measured as the force applied to the material to achieve a deformation of $70 \%$, is used for reference. In early experiments, agar-agar and $\kappa$-carrageenan are tested as the only hydrocolloids in a gel recipe. Although excellent gelling agents in water, they do not work as well in near-saturated $\mathrm{NaI}$ solution. The agar-agar gel does not solidify sufficiently and is severely discoloured, while the use of $\mathrm{k}$-carrageenan (Fig. 2, left) results in very fragile gels which are too delicate to even be transferred to a texture analyser.

The following range of hydrocolloids is tested in all combinations (groups of 2-4): $\kappa$-carrageenan, locust bean
Table 2 Dispersion formulas, refractive indices at 650 and $589 \mathrm{~nm}$ for selected materials

\begin{tabular}{lll}
\hline Material & Dispersion formula & $\begin{array}{l}\text { Refractive index } \\
\text { at } \lambda=650 \mathrm{~nm} \text { and } \\
\lambda=589 \mathrm{~nm}\end{array}$ \\
\hline Water & - & 1.331 \\
& & 1.332 \\
NaI & $n=\sqrt{1+\frac{1.994 \lambda^{2}}{\lambda^{2}-0.176}}$ & {$[29]$} \\
& {$[29]$} & 1.775 \\
Sucrose solution $(30 \%)$ & $n=1.3839-\frac{0.01611}{\lambda^{2}}+\frac{0.00516}{\lambda^{4}}$ & 1.786 \\
& {$[30]$} & 1.345 \\
Sucrose solution & - & 1.352 \\
(45\%) & & 1.402 \\
PMMA & $n=\sqrt{1+\frac{1.1819 \lambda^{2}}{\lambda^{2}-0.011313}}$ & $130]$ \\
& {$[29]$} & 1.488 \\
& & 1.491 \\
\hline
\end{tabular}

Values in italics correspond to the wavelength $\lambda=589 \mathrm{~nm}$ 
Fig. 2 Gel particles made with $0.8 \% \mathrm{w} / \mathrm{w}$ of $\kappa$-Carrageenan (left); gels made according to Table 3 (right)
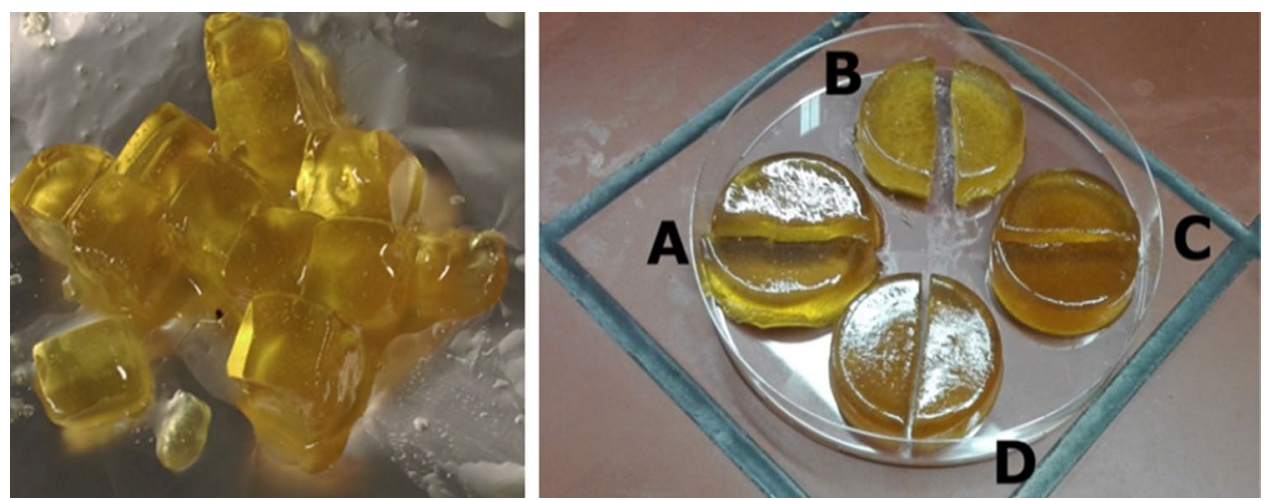

gum, xanthan gum, guar. Agar-agar is tested alone and in combination with $\kappa$-carrageenan and found to be unfit in this context due to intense discoloration, which is significantly stronger than the usual yellowing due to oxidization of $\mathrm{NaI}$ solutions. Gels are prepared by mixing $10 \mathrm{ml}$ of water with $0.1 \mathrm{~g}$ of the respective hydrocolloids in $15 \mathrm{ml}$ plastic reaction tubes. A standard laboratory vortex shaker is used to suspend the solids. The tubes are then treated in an automatic heating shaker at $99{ }^{\circ} \mathrm{C}$ for $2 \mathrm{~min}$. While xanthan and guar are soluble in cold water, higher temperatures are needed to dissolve $\mathrm{K}$-carrageenan $\left(60^{\circ} \mathrm{C}\right)$ and locust bean gum $\left(80^{\circ} \mathrm{C}\right)$ [31]. Based on experience, the continuous shaking and the selected temperature of $99^{\circ} \mathrm{C}$ are sufficient to achieve the necessary temperature within the reaction tubes.

The apparent quality of the resulting gels is evaluated visually by removing them from the tubes. Assessment parameters are transparency, clarity, brightness and firmness. Sufficiently firm gels can only be removed by force with a spatula and then spread out visibly as solid chunks. Insufficiently solid gels can be poured out as a thick, viscous liquid. Most of the hydrocolloids appear to react with iodide ions resulting in a dark purple discoloration similar to a starch-iodide complex.

Ultimately, only a combination of $\kappa$-carrageenan and locust bean gum is able to solidify the NaI solution and sustain its stability. While $\kappa$-carrageenan alone at a concentration of $0.8 \% \mathrm{w} / \mathrm{w}$ does lead to apparent gelation (Fig. 2, left), the structure is not sufficiently stable. Locust bean gum alone increases the viscosity of the solution but forms no solid network.

- Carrageenans are linear sulphated polysaccharides extracted from red seaweeds and composed of alternating $(1,3)$ linked galactose units ( $\beta$-D-galactopyranose and $(1,4)$ linked $\alpha$-D-galactopyranose) and 3,6-anhydro- $\alpha$-Dgalactopyranose. Reportedly, $\kappa$-carrageenan has excellent gelling properties caused by replacement of $\alpha$-Dgalactopyranose by 3,6 -anhydro- $\alpha$-D-galactopyranose. During the gelling process of $\kappa$-carrageenan the single
Table 3 Successfully prepared gels with locust bean gum and $\kappa$-carrageenan

\begin{tabular}{lll}
\hline Gel & Locust bean gum $(\% \mathrm{w} / \mathrm{w})$ & $\begin{array}{l}\kappa-\text { carrageenan } \\
(\% \mathrm{w} / \mathrm{w})\end{array}$ \\
\hline $\mathrm{A}$ & 0.5 & 0.5 \\
$\mathrm{~B}$ & 0.5 & 1.0 \\
$\mathrm{C}$ & 1.0 & 0.5 \\
$\mathrm{D}$ & 1.0 & 1.0 \\
\hline
\end{tabular}

entangled units form the helices, which are connected at the end [32-35].

- Locust bean gum is a polysaccharide made up mostly of a chain of $(1,4)$ linked $\beta$-D-mannopyranose units which contains side chains of $(1,6)$ linked $\alpha$-D-galactopyranose. $20-25 \%$ of the mannose units carry a side chain [36].

In the following experimental stage, gels are prepared with $\kappa$-carrageenan and locust bean gum in concentrations of $0.5-1.0 \% \mathrm{w} / \mathrm{w}$ for each ingredient as listed in Table 3 . The hydrocolloids are mixed with the same $\mathrm{NaI}$ solution as before in $50 \mathrm{ml}$-plastic reaction vessels, heated to $99^{\circ} \mathrm{C}$ for two minutes and then poured into glass dishes. These were left to cool to room temperature and then kept in a refrigerator $\left(3{ }^{\circ} \mathrm{C}\right)$ overnight (Fig. 2, right). Before further analysis, they are taken out and left at room temperature for $30 \mathrm{~min}$. Texture analysis is carried out at room temperature, but without temperature control. Refractive indices of the liquids, gels and acrylic glass involved are initially looked up in the Refractive Index Info database [29] and measured using a standard Abbe refractometer at $589 \mathrm{~nm}$. The density of the particles is assumed to be almost exactly the same as that of $\mathrm{NaI}$ solution, as the amount of mass added is very low and no significant change of volume is observed. From the formulation of gel $\mathrm{D}$, the increase in density can be estimated as about $2 \%$ (to $1890 \mathrm{~kg} / \mathrm{m}^{3}$ from $1850 \mathrm{~kg} / \mathrm{m}^{3}$ as mentioned in Table 1). The yellowing of the gels due to oxidization is not stronger than that of liquid $\mathrm{NaI}$ solution and does not decrease their transparency. 


\section{Results and discussion}

The cubic particles shown on the left of Fig. 2 appear to be solid but are too fragile to even transfer them to a texture analyser. Their cubic shapes $(\mathrm{d}=8 \mathrm{~mm})$ result from a larger silicone mould used in an early experimental stage where several different formulations are scanned. Materials on the right of Fig. 2, with added locust bean gum, are cast into glass petri dishes $(\mathrm{d}=18 \mathrm{~mm})$ instead to facilitate texture analysis. In a first assessment of their apparent mechanical stability, they can be cleanly cut with a knife. Their mechanical resistance is measured in a texture analyser with a simple penetration test.

While it could be expected that sucrose would quickly diffuse into the gel, changing its composition and thereby its refractive index, both the liquid and the gel phase are in a nearly saturated state which appears to offer no sufficient electrochemical potential difference. Two years after producing the gel and storing it in the described NaI-sucrose solution, its refractive index has not changed.

\subsection{Mechanical resistance}

Resistance against deformation is measured and compared to that of diced apples (with an average length of the edge of $15 \mathrm{~mm}$ at room temperature, $30{ }^{\circ} \mathrm{C}$ and $50{ }^{\circ} \mathrm{C}$. RIM gels are only measured at room temperature in a cylindrical disc shape of $18 \mathrm{~mm}$ average diameter and $15 \mathrm{~mm}$ average thickness. A circular disc-shaped deformation tool $(\mathrm{d}=40 \mathrm{~mm})$ is used. The disc moves towards the particle at an initial velocity of $1 \mathrm{~mm} / \mathrm{s}$ until a force of $0.1 \mathrm{~N}$ is measured, which indicates contact with the particle. A velocity of $0.5 \mathrm{~mm} / \mathrm{s}$ is then applied downwards and force is measured over distance travelled. The force required to deform the material increases, reaches a maximum and decays when the structure of the gel disintegrates irreversibly. The exact distance travelled as well as compression until the point of maximum force is reached varies due to slight inhomogeneity of the samples. The test is stopped automatically at a deformation rate of the material of $70 \%$. Figure 3 reports only the maximum force value measured and compares it to that of diced apples at three different temperatures analysed the same way. The comparison shows that the behaviour of the most rigid Gels C and D in the same range as diced apples between 30 and $50{ }^{\circ} \mathrm{C}$. Based on this similarity, their behaviour in a stirred suspension is expected to be similar to that of diced apples at these temperatures.

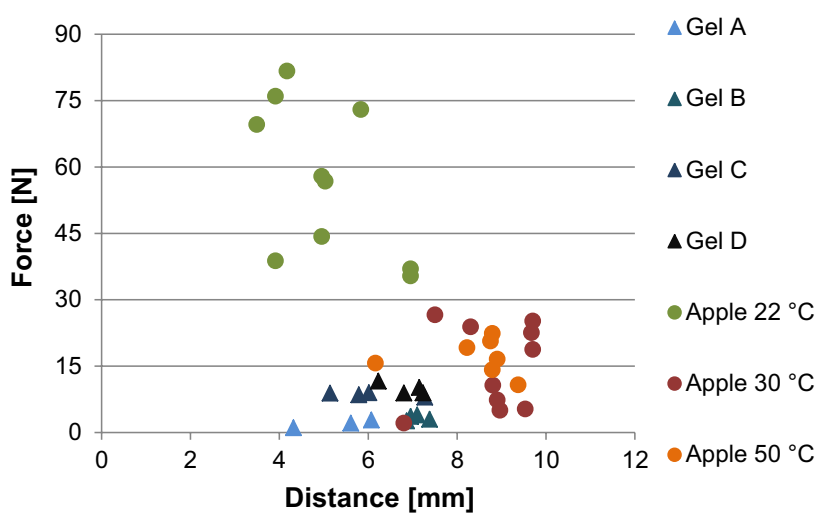

Fig. 3 Strength of gels made according to Table 3 (triangles) compared to the strength of diced apples at different temperatures (circles)

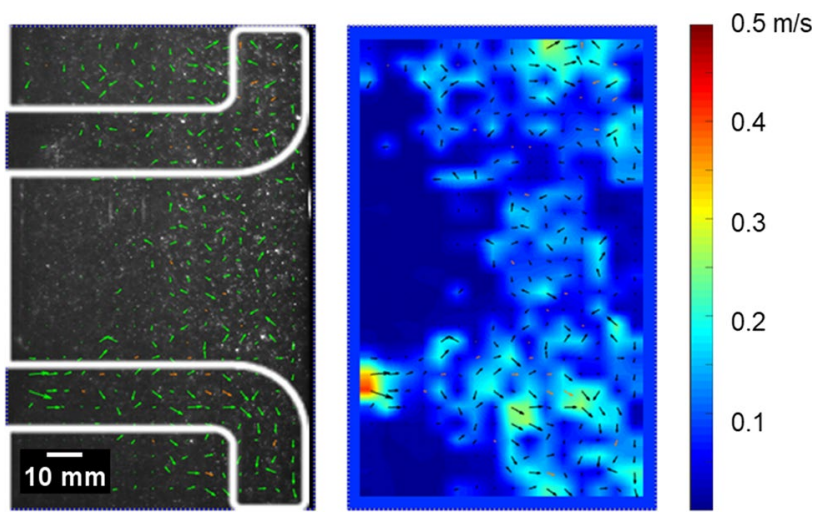

Fig. 4 Stirring of NaI solution with a $10 \%$ w/w particle load at $30 \mathrm{rpm}$

\subsection{Stirring experiments}

To put the developed material to the practical test, stirring experiments are conducted with the transparent apparatus described above and depicted in Fig. 1 (the region of interest is marked by a red rectangle). Gel $\mathrm{D}$ according to Table 1 is cast and passed through a sieve of $2 \mathrm{~mm}$ mesh width. The gel particles are suspended in NaI solution with and without sucrose according to Table 1. PMMA tracer particles with an average diameter of $50 \mu \mathrm{m}$ are suspended in the solutions to enable flow visualization as necessary for the application of PIV evaluation methods.

The stirring vessel is illuminated with a red $(650 \mathrm{~nm})$ line laser $(100 \mathrm{~mW})$ pointed through the axes of both the container and the stirrer, penetrating the whole width of the vessel but covering its full height only on the side opposite to the line of entry, where the region of interest is located. Images are recorded at a rate of 60 frames per second due to data capacity limitations. This is not a sufficiently fine resolution to conduct conventional PIV, but was applied here to show the applicability of the material. The region of interest is marked in Fig. 1 with a red rectangle and in 

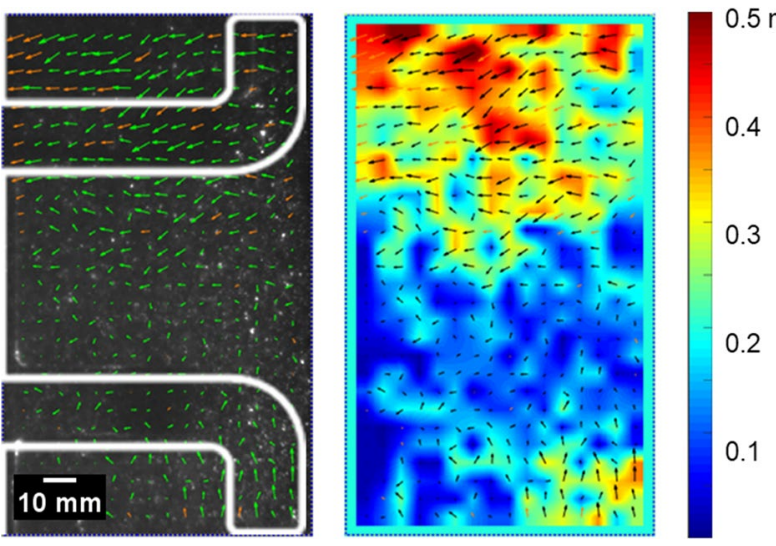

Fig. 5 Stirring of NaI-sucrose solution with a $50 \%$ w/w particle load at $90 \mathrm{rpm}$

Figs. 4 and 5 with the outline of the stirrer in white. The figures show the stirrer position one sixtieth of a second before passing through the illuminated plane. Using PIVlab, the PIV module for MATLAB [37, 38], images are analysed in three iterations with the size of the interrogation windows decreasing from 128 over 64 down to 32 pixels and the search windows from 64 over 32 down to 16 pixels respectively. Statistical vector validation is applied using a filter with the sevenfold standard deviation and a local median filter with an initial residual of 5 and a threshold of $\varepsilon=0.25$ (PIVlab default settings).

Figures 4 and 5 show examples of the resulting measurement data in the form of vector fields (left) and velocity magnitude (right) distributions evaluated from PIV snapshots (individual pairs of consecutive images). For all three image pairs, the stirrer is at the same position relative to the camera. Colour bars on the far right are only valid for velocity magnitude plots. The stirrer is still visible (Fig. 5) because the edges are not manufactured perfectly smooth, causing visible light scattering. It leads to artefacts at the left edges of Figs. 4 and 5 where the reflection in the corner of the stirrer is falsely detected as motion of the fluid. This can be an even more serious problem when more three-dimensional stirrer designs are used.

Addition of the gel particles to the flow, their visibly successful suspension in the liquid, recording of PIV imagery and the evaluation of the recorded images show the applicability of the developed material in this area of research. Fluid motion across the full range of the stirred volume and the immediate surroundings of the stirrer could not be observed without the successful refractive index matching of the acrylic glass, stirred liquid and suspended particle phases. While the mechanical resistance of the developed gels matches that of apple matter in a simple texture analytical assessment, biological matter is an anisotropic and inhomogeneous material which can behave very differently depending on the direction and form of stress exerted onto it. However, the materials are useful for the analysis of stirring flows as described.

\section{Conclusions and outlook}

A model material system for deformable solid materials is produced as hydrocolloid gels and their refractive index are adjusted by adding a substantial amount of NaI; out of the hydrocolloids considered here, this will work for $\kappa$-carrageenan and a combination of locust bean gum with $\kappa$-carrageenan. With these gels, the mechanical firmness (but not the resistance against breaking) of soft granular matter can be mimicked and the movement of gel particles in suspensions as well as the induced stress, the resulting deformation and ultimately, the inflicted damage can be observed with optical methods. The rigidity of the gels may be varied to some extent with the concentrations of the respective hydrocolloids, which, at low concentrations, have no apparent effect on the refractive index. Further texture analytics will be carried out to investigate the applicability of the presented material to model other soft granular matter (vegetables, meat, and curd) or to model material structures in more detail, e.g. different layers of tissue or sediment. Extraction processes (coffee, tea, gelatine) are another conceivable application, where excessive particle disintegration can reduce the efficiency of the subsequent separation process. Jamming transitions as well as jammed systems, which typically occur at higher particle volume fractions $(80 \% \mathrm{w} / \mathrm{w}$ and more) than considered here could be optically analysed [1-3], if the viscosities of the matching liquids can be adjusted to the specific jamming problem. As part of a validation method, this could strongly benefit especially the computational results in this area. Introducing ethanol, thickeners or other components may yield more degrees of freedom in modelling their flow behaviour.

Acknowledgements Open Access funding provided by Projekt DEAL. This IGF (German Federation of Industrial Research Associations) Project of the FEI (Research Association of the German Food Industry) was supported via AiF (German Federation of Industrial Research Associations) within the programme for promoting the Industrial Collective Research (IGF) of the German Ministry of Economic Affairs and Energy (BMWi), based on a resolution of the German Parliament. Project AiF 19011 N.

\section{Compliance with ethical standards}

Conflict of interest The authors declare that they have no conflict of interest.

Open Access This article is licensed under a Creative Commons Attribution 4.0 International License, which permits use, sharing, adaptation, distribution and reproduction in any medium or format, as long as you give appropriate credit to the original author(s) and the source, provide a link to the Creative Commons licence, and indicate if changes were made. The images or other third party material in this article are included in the article's Creative Commons licence, unless indicated 
otherwise in a credit line to the material. If material is not included in the article's Creative Commons licence and your intended use is not permitted by statutory regulation or exceeds the permitted use, you will need to obtain permission directly from the copyright holder. To view a copy of this licence, visit http://creativecommons.org/licenses/by/4.0/.

\section{References}

1. van Hecke, M.: Jamming of soft particles: geometry, mechanics, scaling and isostaticity. J. Phys.: Condens. Matter 22, (2010)

2. Zhang, H.P., Makse, H.A.: Jamming transition in emulsions and granular materials. Phys. Rev. 72, (2005)

3. Boromand, A., et al.: The role of deformability in determining the structural and mechanical properties of bubbles and emulsions. Soft Matter 15, (2019)

4. Dabiri, J.O., et al.: Flow patterns generated by oblate medusan jellyfish: field measurements and laboratory analyses. J. Exp. Biol. 208, 1257-1265 (2005)

5. Vettori, D., Nikora, V.: Flow-seaweed interactions: a laboratory study using blade models. Environ. Fluid Mech. 18, 611-636 (2017)

6. Govindarajan, V., et al.: Impact of tissue factor localization on blood clot structure and resistance under venous shear. Biophys. J. 114, 978-991 (2018)

7. Zima-Kulisiewicz, B. E.: Einfluss von fluiddynamischen Effekten auf granularen Belebtschlamm. Erlangen: Dissertation, Universität Erlangen-Nürnberg (2008)

8. Díez, L., et al.: Investigation of multiphase flow in sequencing batch reactor (SBR) by means of hybrid methods. Chem. Eng. Sci. 62, 1803-1813 (2007)

9. Díez Robles, L.: Novel Hybrid Methods Applied for the Numerical Simulation of Three-Phase Biotechnological Flows. [Dissertation]. Erlangen: Universität Erlangen-Nürnberg (2009)

10. Budwig, R.: Refractive index matching methods for liquid flow investigations. Exp. Fluids 17, 350-355 (1994)

11. Bai, K., Katz, J.: On the refractive index of sodium iodide solutions for index matching in PIV. Exp. Fluids 55, 1704 (2014)

12. Kraume, M.: Mischen und Rühren: Grundlagen und moderne Verfahren. Wiley-VCH, Weinheim (2003)

13. Kraume, M.: The development of stirring technology from an empirical art to science. ChemBioEng Rev. 2(4), 279-289 (2015)

14. Liu, G., et al.: A comparative experimental study of the anaerobic treatment of food wastes using an anaerobic digester with a polyamide stirring rake or a stainless-steel stirring rake. J. Environ. Manag. 218, 435-441 (2018)

15. Lia, W., et al.: Effect of stirring rate on microstructure and properties of microporous mullite ceramics. J. Mater. Process. Technol. 261, 159-163 (2018)

16. Peiter, A.S., et al.: Stirring and mixing in ethylic biodiesel production. J. King Saud Univ. Sci. 32, 54-59 (2018)

17. Raffel, M., et al.: Particle Image Velocimetry: A Practical Guide. Springer, Berlin (2007)

18. Iskander, M.: Modelling with Transparent Soils. Springer, Berlin (2010). ISBN 978-3-642-02500-6

19. Dijksman, J.A., et al.: Refractive index matched scanning of dense granular materials. Rev. Sci. Instrum. 83, 011301 (2012)
20. Cui, M.M., Adrian, R.J.: Refractive index matching and marking methods for highly concentrated solid-liquid flows. Exp. Fluids 22, 261-264 (1994)

21. Daviero, G., Roberts, P.J., Maile, K.: Refractive index matching in large-scale stratified experiments. Exp. Fluids 31, 119-126 (2001)

22. Dijksman, J.A., Brodu, N., Behringer, R.P.: Refractive index matched scanning and detection of soft particles. Rev. Sci. Instrum. 88, 051807 (2017)

23. Byron, M.L., Variano, E.A.: Refractive-index-matched hydrogel materials for measuring flow-structure interactions. Exp. Fluids 54, 1456 (2013)

24. Franklin, J., Wang, Z.Y.: Refractive index matching: a general method for enhancing the optical clarity of a hydrogel matrix. Chem. Mater. 14, 4487-4489 (2002)

25. Weitzman, J.S., et al.: On the use of refractive-index-matched hydrogel for fluid velocity measurement within and around geometrically complex solid obstructions. Exp. Fluids 55, 1862 (2014)

26. Buchanan, C.F., et al.: Three-dimensional microfluidic collagen hydrogels for investigating flow-mediated tumor-endothelial signaling and vascular organization. Tissue Eng. Part C 20(1), 64-75 (2014)

27. Wiederseiner, S., et al.: Refractive-index and density matching in concentrated particle suspensions: a review. Exp. Fluids 50, 1183-1206 (2011)

28. Cardarelli, F.: Properties of Materials. Materials Handbook: A Concise Desktop Reference. Springer, Cham (2018)

29. Polyanskiy, M.: Refractive Index Info. https://refractiveindex.info/ (2017). Accessed 15 May 2017

30. Belay, A., Assefa, G.: Concentration, wavelength and temperature dependent refractive index of sugar solutions and methods of determination contents of sugar in soft drink beverages using laser lights. J. Lasers Opt. Photonics 5(2), 1000187 (2018)

31. Wüstenberg, T.: Cellulose and Cellulose Derivatives in the Food Industry. Wiley-VCH, Weinheim (2014)

32. Rochas, C., Rinaudo, M.: Mechanism of gel formation in k-Carrageenan. Biopolymers 23, 735-745 (1984)

33. Yu, G., et al.: Structural studies on k-Carrageenan derived oligosaccharides. Carbohydr. Res. 337, 433-440 (2002)

34. Elgobashi, S.: An Updated Classification Map of of ParticleLaden Turbulent Flows. In: IUTAM Symposium on Computational Approaches to Multiphase Flow. Springer, Dordrecht (2006)

35. Campo, V.L., et al.: Carrageenans: biological properties, chemical modifications and structural analysis - a review. Carbohydr. Polym. 77, 167-180 (2009)

36. Belitz, H.-D., Grosch, W., Schieberle, P.: Lehrbuch der Lebensmittelchemie. Springer, Berlin (2008)

37. Thielicke, W., Stamhuis, E.J.: PIVlab-Time-Resolved Digital Particle Image Velocimetry Tool for MATALB. Latest version v1.4: s.n., 12.02.2016

38. MATLAB: MATLAB and Image Processing Toolbox Release 2016b. Natick. The Mathworks Inc., Massachusetts (2016)

Publisher's Note Springer Nature remains neutral with regard to jurisdictional claims in published maps and institutional affiliations. 